\title{
A Geometric Approach to Inference in Set-Identified Entry Games
}

\author{
Christian Bontemps* Rohit Kumar ${ }^{\dagger}$
}

December 2019

\begin{abstract}
In this paper, we consider inference procedures for entry games with complete information. Due to the presence of multiple equilibria, we know that such a model may be set-identified without imposing further restrictions. We complete the model with the unknown selection mechanism and characterize geometrically the set of predicted choice probabilities, in our case, a convex polytope with many facets. Testing whether a parameter belongs to the identified set is equivalent to testing whether the true choice probability vector belongs to this convex set. Using tools from the convex analysis, we calculate the support function and the extreme points. The calculation yields a finite number of inequalities, when the explanatory variables are discrete, and we characterized them once for all. We also propose a procedure that selects the moment inequalities without having to evaluate all of them. This procedure is computationally feasible for any number of players and is based on the geometry of the set. Furthermore, we exploit the specific structure of the test statistic used to test whether a point belongs to a convex set to propose the calculation of critical values that are computed once and independent of the value of the parameter tested, which drastically improves the calculation time. Simulations in a separate section suggest that our procedure performs well compared with existing methods.
\end{abstract}

Keywords: set-identification, entry games, convex set, support function

\footnotetext{
${ }^{*}$ Corresponding author. ENAC \& Toulouse School of Economics, Université de Toulouse, 1, Esplanade de l'université, 31080 Toulouse cedex 06, France, email: christian.bontemps@tse-fr.eu.

†Indian Statistical Institute (ISI), 7 S.J.S Sansanwal Marg, New Delhi 110016, India, email: sirohi.rohit@gmail.com
} 


\section{Introduction}

This paper provides an estimation procedure for empirical models of entry and market structure, also called entry games, which may be set-identified. Entry games are very popular in the empirical Industrial Organization literature because they allow researchers to study the nature of firms' profits and the nature of competition between firms from data that are generally easy to collect. They were popularized by the seminal works of Bresnahan and Reiss (1991a), Bresnahan and Reiss (1991b) and Berry (1992). ${ }^{1}$ However, the econometric analysis of entry games is complicated by the presence of multiple equilibria, a problem that affects the standard estimation strategy. Without additional assumptions, the model is indeed incomplete. Various solutions have been proposed in the literature. First, assumptions can be added regarding the (unknown) selection mechanism in regions of multiple equilibria. Reiss (1996) considers a specific order of entry, and Bjorn and Vuong (1984), randomly draw the equilibrium selection. Bajari et al. (2010) introduce a parametric specification of the selection mechanism, and Grieco (2014) extends it to a non-parametric function of observables and non-observables. Alternatively, it is sometimes possible to estimate an entry game from the observation of some outcome that is independent of the true selection mechanism. In their seminal work with heterogeneous firms, Bresnahan and Reiss (1991b) report that the model uniquely predicts the number of active firms. Berry (1992) generalizes the estimation of the profit function from the observed number of active firms for more than two players. A tremendous number of empirical applications have followed this path (see the survey by de Paula (2012)), notably including Mazzeo (2002), Reiss (1996), Cleeren et al. (2010), and Sampaio (2007), among others. However, adding assumptions could lead to a misspecified model, and working on certain invariant outcomes may lead to a suboptimal procedure (see Tamer (2003)). The recent and blossoming literature on partial/set-identification, following earlier work by Manski (1995), makes it possible to estimate a model that does not uniquely predict actions by using bounds. Following Tamer (2003), the presence of multiple equilibria does not imply partial identification, ${ }^{2}$ but the literature provides inference methods that are eligible in both cases. Ciliberto and Tamer (2009)

\footnotetext{
${ }^{1}$ See Berry and Reiss (2007) for a survey.

${ }^{2}$ However, identification at infinity, as in Tamer (2003), may lead to inference procedures that are non standard; see Khan and Tamer (2010).
} 
were the first to use bounds to estimate an entry game with complete information.

In this paper, we complete the model with the selection mechanism, $\eta(\cdot)$, and characterize the set of predicted choice probabilities generated by the variation of $\eta(\cdot)$ in the space of admissible selection mechanisms. Our first contribution is to characterize more deeply the geometric structure of this set. The set is a convex polytope with many facets (because we focus on pure strategy Nash equilibria), and the number of facets increases exponentially with the number of players. Alternative equilibrium concepts have been proposed in the literature (as in Aradillas-Lopez and Tamer (2008), Beresteanu et al. (2011) or Galichon and Henry (2011)). Changing the equilibrium concept affects some of the calculations provided in this paper and, sometimes, increases the complexity but does not alter the general philosophy. Moreover, Nash equilibrium is the most commonly used solution concept. In this paper, we derive a closed-form expression for the support function of this polytope, the extreme points (or vertices) of which can also be calculated as a function of the primitives of the model. These vertices are indeed characterized by an order of outcome selection in the regions of multiple equilibria. Each vertex is also geometrically defined by the intersection of some supporting hyperplanes. We are able to define the cone of outer normal vectors of these hyperplanes and, thereby, the inequalities that are binding in this point.

Testing whether a parameter belongs to the identified set is equivalent to testing whether the true choice probability vector belongs to this convex set. Following Rockafellar (1970), the support function defines a continuum of inequalities that have to be satisfied for any point in the set. This characterization has already been used in the set-identification literature by, in particular, Beresteanu and Molinari (2008), Beresteanu et al. (2011) and Bontemps et al. (2012). Geometrically, the support function in a given direction leads to an inequality that detects whether the point of interest belongs to the same halfspace than the convex set itself. This continuum of inequalities simplifies here toward a finite number because of the specific structure of the entry game considered in this paper.

However, when the number of players increases, the number of facets of the polytope increases exponentially, and, therefore, the smallest number of inequalities necessary to have a sharp characterization - from 16 in a game with three players to more than one million in a game with six players. The standard approach for moment inequality models is to first evaluate all the moments, 
then eliminate the ones that are far from being binding (see Andrews and Soares, 2010, for the unconditional case). Our second contribution is to provide a geometric selection procedure that does not require us to evaluate all the moments and for which the computational cost is polynomial in the dimension of the outcome space $\left(2^{N}\right)$. The idea is to recursively identify the extreme point of the set that is the most relevant for demonstrating that our point, the true choice probability vector, belongs to the polytope. We then test only the inequalities related to the facets of this extreme point and do not evaluate the other inequalities. The number of relevant moments grows at the rate $2^{N}$ as in Ciliberto and Tamer (2009), but with a sharper characterization of the set. This is a considerable improvement in terms of computational burden with respect to alternative sharp characterization methods (such as the two step approaches of Andrews and Soares (2010) and Romano et al. (2014)).

Furthermore, and more importantly, we develop a test procedure for the hypothesis of whether the true choice probability vector belongs to this polytope that exploits the specific structure of the test statistic. When we test whether a parameter $\theta$ belongs to the identified set, the feasible test statistic depends on the support function of the polytope, which is fixed, and the choice probability vector, which is estimated. The asymptotic distribution depends on $\theta$ in a specific manner. It allows us to derive critical values for our test procedures that are calculated once and valid for any $\theta$. This is a tremendous simplification with respect to a general moment inequality model. Some of the recommended test procedures can be conservative, but, one, which exploits the maximum number of facets at any extreme point, is not. This maximum number can be determined by brute force using the geometric structure of the set; we also provide an upper bound. Simulations highlight that our method works well with the sample sizes that are usually considered in the empirical literature (from 500 to 2000 observations) and outperforms existing inference procedures.

This paper belongs to the growing literature on set-identification and lies at the intersection between the moment inequality literature and the literature on set-identification that exploits the geometric structure. A model is generally set-identified when the data are incomplete because of some missing information (a censorship mechanism, two-sample combination, or multiple equilibria). In entry games, the unknown selection mechanism in regions of multiple equilibria is the missing one, but it is naturally bounded between 0 and 1 . These bounds lead to moment inequalities (see, 
for example, Ciliberto and Tamer (2009)). Moment inequality procedures are now well developed in the partial identification literature, which includes contributions by, among others, Chernozhukov et al. (2007), Rosen (2008), Andrews and Soares (2010), Bugni (2010), Canay (2010), Romano and Shaikh (2008, 2010, 2012), Chernozhukov et al. (2015), Andrews and Shi (2013) or AradillasLopez and Rosen (2017). However, in most cases, the structure of the missing information is not exploited. Other contributions exploit this missing information by characterizing the identified set directly or indirectly through a convex set, and, then, through the support function. The support function provides a collection of moment inequalities, but these moment inequalities have a particular structure and are exploited differently than in the general moment inequality literature (see, in particular, the two surveys by Molchanov and Molinari (2015) and Bontemps and Magnac (2017)).

Papers that have exploited the structure of the missing information either use random set theory (introduced in econometrics by Beresteanu and Molinari, 2008), optimal transport (Galichon and Henry (2011)) or complete the model, as in Bontemps et al. (2012). Fundamentally, all methods are intended to provide a sharp characterization of the identified set and lead to a collection of moment inequalities that are specifically determined using the tools of the approach considered. They all lead to the same set of inequalities. In this spirit, they can be seen as equivalent. Redundant ones are eliminated through the characterization of what Galichon and Henry (2011) call the core determining class. In our case, we also characterize the core determining class, i.e. the collection of facets of the convex set, by a necessary and sufficient condition. However, in addition to these equivalences, our geometric approach is able to select the subsets of moments that are close to binding without having to evaluate all of them and to determine the maximum number of moments that are binding.

Section 2 considers the general entry game with $N$ players and no explanatory variable. Explanatory variables do not play a specific role in the procedure. We characterize the regions with multiple equilibria and compute the support function of the polytope, i.e., the set of predicted choice probability vectors for a given parameter value. The sequence of inequalities that need to be verified is characterized. The aim of Section 3 is to more deeply exploit the geometry of the polytope to propose a more efficient estimation procedure. We first provide a necessary and suffi- 
cient condition for eliminating any redundant inequalities. We then propose our geometric selection procedure which consists of determining the local extreme point of the polytope and only testing the inequalities relative to the facets at this extreme point. Section 4 calculates the asymptotic distribution of the test statistic used and proposes different calculations of the critical value that are computed once for all. Section 5 proposes a few Monte Carlo simulations to assess the performance of our inference procedure and compare it with existing procedures. Section 6 considers the case with discrete explanatory variables, and Section 7 concludes the paper. The Supplementary Material contains the proofs and the algorithms, the specific details for the three player game and an additional Monte Carlo analysis.

\section{Entry game with $N$ players}

We formalize the entry game with $N$ players/firms. For exposition purposes, we first consider a model without explanatory variables and then extend it in section 6 . We first define some notations before characterizing the identified set.

\subsection{Setup and notations}

\subsubsection{The model}

Let $N$ denote the total number of firms that can enter any market. Following Berry (1992), we introduce a model of market structure where the profit function $\pi_{i m}$ of firm $i$ in a market $m$ is assumed to be independent of the identity of the firm's competitors. All firms decide simultaneously whether to enter the market (the action is $a_{i m}=1$ ) if, in a pure strategy Nash equilibrium, their profit is positive $\left(\pi_{i m}>0\right)$. Otherwise, $\pi_{i m}<=0$, and the action is $a_{i m}=0$. The profit function is assumed, without loss of generality, to be linear in the explanatory variables ${ }^{3}$

$$
\begin{aligned}
& \pi_{i m}=\beta_{i}+\alpha_{i}\left(\sum_{j \neq i} a_{j m}\right)+\varepsilon_{i m} \\
& a_{i m}=\mathbf{1}\left\{\pi_{i m}>0\right\}
\end{aligned}
$$

\footnotetext{
${ }^{3}$ Any (separable) parametric form $\pi_{i m}=f_{i}\left(\sum_{j \neq i} a_{j m} ; \alpha\right)+\varepsilon_{i m}$ can be considered as long as the function $f_{i}(\cdot ; \theta)$ is strictly decreasing in its first argument.
} 
Following the literature on entry games, we assume that $\alpha_{i}<0$, i.e., the presence of more competitors decreases a firm's profit. ${ }^{4}$ The unobserved components $\varepsilon_{i m}, i=1, \ldots, N$ are drawn from a known distribution (up to some parameter vector $\gamma$ ). The econometrician does not observe their values, whereas all firms within a market observe them before deciding whether to enter (complete information case).

For identification, we first need a scale normalization, and thus, we assume that the variance of each shock $\varepsilon_{i m}$ is equal to unity. We denote by $F(\cdot ; \gamma)$ the cumulative distribution function of $\varepsilon_{m}=\left(\varepsilon_{1 m}, \ldots, \varepsilon_{N m}\right)^{\top}$, and we assume that the distribution is continuous with full support. Henceforth, we use the notation $\theta$ for all the parameters in the model $\left(\theta \in \Theta \subseteq \mathbb{R}^{l}\right),{ }^{5}$ and we omit the subscript $m$ for notational convenience.

\subsubsection{The multiplicity of pure strategy Nash equilibria}

For a given market, an outcome $y$ is the vector of actions (in $\{0,1\}^{N}$ ) taken by the firms. There are obviously $2^{N}$ possible outcomes from $(0, \ldots, 0)^{\top}$ to $(1, \ldots, 1)^{\top}$. We denote by $\mathcal{Y}$ this set of possible outcomes. $\mathcal{Y}_{K}$ denotes the subset of outcomes with $K$ active firms in equilibrium, i.e. any $K$ firms playing action 1 . There is 1 outcome with 0 active firms, $N$ outcomes with 1 active firm and $d_{K}=\left(\begin{array}{l}N \\ K\end{array}\right)$ outcomes with $K$ active firms for $K \leq N$. For each $K$, we label the outcomes as $y_{j}^{(K)}, j=1, \ldots, d_{K}$ according to a predefined order. ${ }^{6}$ Globally, we order the outcomes in $\mathcal{Y}$ first by their number of active firms, then according to the predefined order within each $\mathcal{Y}_{K}$ :

$$
\mathcal{Y}=\{\underbrace{y_{1}^{(0)}}_{\mathcal{Y}_{0}}, \underbrace{y_{1}^{(1)}, \ldots, y_{d_{1}}^{(1)}}_{\mathcal{Y}_{1}}, \ldots, \underbrace{y_{1}^{(K)}, \ldots, y_{d_{K}}^{(K)}}_{\mathcal{Y}_{K}}, \ldots, \underbrace{y_{1}^{(N)}}_{\mathcal{Y}_{N}}\} .
$$

It is well known that the model has multiple equilibria, i.e., there are regions of realizations of $\varepsilon$ in which we cannot uniquely predict each firm's action. Consequently, there is no one-to-one mapping between the collection of possible outcomes and the regions of $\varepsilon$ given any parameter value $\theta$.

What is missing from the model is the selection of a given equilibrium in the regions of multiple equilibria. We define this selection mechanism $\eta(\cdot)$ as in Definition 2 of Galichon and Henry (2011).

\footnotetext{
${ }^{4}$ The case in which $\alpha_{i}>0$ could be handled equivalently. Gualdani (2019) considers, in a network formation game, these two cases.

${ }^{5} \theta=\left(\beta_{1}, \ldots, \beta_{N}, \alpha_{1}, \ldots, \alpha_{N}, \gamma\right)^{\top}$

${ }^{6}$ The order for $K=1$ is $\left((1,0, \ldots, 0)^{\top},(0,1,0 \ldots, 0)^{\top} \ldots(0, \ldots, 0,1)^{\top}\right)$, and so forth.
} 
Definition 1 (Equilibrium selection mechanism) An equilibrium selection mechanism is a conditional probability $\eta(\cdot \mid \varepsilon ; \theta)$ of y given $\varepsilon$ such that the selected value of the outcome variable is actually an equilibrium predicted by the game.

We denote by $\mathcal{E}$ the set of selection mechanisms and by $P(\theta, \eta)$ the predicted choice probability vector when the parameter of the model is $\theta$ and the selection mechanism is $\eta(\cdot)$. We partition this vector according to the partition of $\mathcal{Y}$ as $^{7}$

$$
P(\theta, \eta)=(\underbrace{P_{1}^{(0)}(\theta, \eta)}_{P^{(0)}(\theta, \eta)}, \ldots, \underbrace{P_{1}^{(K)}(\theta, \eta), \ldots, P_{d_{K}}^{(K)}(\theta, \eta)}_{P^{(K)}(\theta, \eta)}, \ldots, \underbrace{P_{1}^{(N)}(\theta, \eta)}_{P^{(N)}(\theta, \eta)})^{\top} .
$$

One solution to the multiple equilibria problem consists of making assumption on this selection mechanism like in Reiss (1996) or Cleeren et al. (2010), for example. The vector of predicted choice probabilities is a point in $[0,1]^{2^{N}}$ and standard inference techniques can be used. This is, of course, ad hoc and may lead to misspecification.

Another solution, ours, following the literature on set-identification, consists of characterizing all the possible choice probabilities predicted by the model. The vector of predicted choice probabilities, instead of being a point, belongs to a (convex) set that we characterize. Different sets of values $(\theta, \eta)$ may generate the same point $P(\theta, \eta) .{ }^{8}$ Our goal is to characterize the ones which generate the true choice probability vector. In the next subsection, we first characterize the set of choice probabilities predicted by the model.

\subsection{From the set of choice probabilities to the identified set}

In this section, we want to characterize the set of predicted choice probabilities. To do so, we need to understand the multiplicity structure and characterize it. Then, we derive a parametrization of the set.

\footnotetext{
${ }^{7}$ In particular, $P_{i}^{(K)}(\theta, \eta)$ denotes $\operatorname{Prob}\left(y=y_{i}^{(K)} \mid \theta, \eta\right)$.

${ }^{8}$ Observe that having multiple equilibria does not automatically guarantee to have set/partial identification. In the following, our statistical procedure, which consists of inverting a test, is valid for point or set-identified models.
} 


\subsubsection{The regions of multiple equilibria}

Our specification ensures that multiple equilibria only involve outcomes with the same number of active firms, i.e., within $\mathcal{Y}_{K} \cdot{ }^{9}$ Therefore, we focus on subsets of outcomes $S \subseteq \mathcal{Y}_{K}$ to characterize the multiple equilibria regions. We say that a subset $S \subseteq \mathcal{Y}_{K}$ is in multiplicity if the prediction of the game is all outcomes in $S$ and no outcome outside $S$ for $\varepsilon$ in a non empty set, $\mathcal{R}_{S}^{(K)}(\theta)$. $\mathcal{R}_{S}^{(K)}(\theta)$ is called a multiple equilibria region. We denote by $S^{(K)}$ the collection of subsets $S$ of $\mathcal{Y}_{K}$ in multiplicity. ${ }^{10}$

$$
S^{(K)}=\left\{S \subseteq \mathcal{Y}_{K}:|S| \geq 2 \text { and } S \text { is in multiplicity }\right\} .
$$

Note that not all subsets of cardinality greater than two are elements of $S^{(K)}$. For example, when $N=4$ and $K=2, S_{1}=\left\{(1,1,0,0)^{\top},(0,0,1,1)^{\top}\right\}$ is not in multiplicity whereas the subset $S_{2}=\left\{(1,1,0,0)^{\top},(1,0,1,0)^{\top}\right\}$ is.

We now present a necessary and sufficient condition for $S$ to be in multiplicity. Define $N_{0}$ (resp. $N_{1}$ ) the set of indices of firms that always play action 0 (resp. 1) across $S$. $n_{0}$ and $n_{1}$ are their cardinalities. $N_{0}$ and $N_{1}$ being fixed, there are $\left(\begin{array}{c}N-n_{0}-n_{1} \\ K-n_{1}\end{array}\right)$ possible outcomes in $\mathcal{Y}_{K}$ corresponding to the remaining choice of the $K-n_{1}$ firms which play action 1 among the $N-n_{0}-n_{1}$ remaining ones. $S$ should contain all these possibilities to be in multiple equilibria and it is formalized in the next proposition.

Proposition 1 A set $S \subseteq \mathcal{Y}_{K}$ is in multiplicity if and only if $|S|=\left(\begin{array}{c}N-n_{0}-n_{1} \\ K-n_{1}\end{array}\right)$.

Observe that, for our particular examples above, $S_{1}$ is not in multiplicity because $n_{0}=n_{1}=$ 0 and, consequently, the subset should contain $\left(\begin{array}{l}4 \\ 2\end{array}\right)=6$ outcomes with two active firms to be in multiplicity. $\quad S_{2}$ is in multiplicity because $n_{0}=n_{1}=1$ and it collects all possible outcomes $\left(\left(\begin{array}{c}4-1-1 \\ 2-1\end{array}\right)=2\right)$. The proof of Proposition 1 characterizes also the region $\mathcal{R}_{S}^{(K)}(\theta)$ of $\varepsilon$.

Following Proposition 1, we count the number of multiple equilibria regions.

\footnotetext{
${ }^{9}$ See the proof of Proposition 1 in the Supplementary Appendix.

${ }^{10}$ Note that the maximum number of such subsets is equal to $2^{d_{K}}-d_{K}-1$.
} 
Proposition 2 The cardinality of $S^{(K)}$, i.e., the number of multiple equilibria regions predicting $K$ active firms, for $1 \leq K \leq N-1$ is equal to

$$
\left|S^{(K)}\right|=\sum_{n_{1}=0}^{K-1} \sum_{n_{0}=0}^{N-K-1}\left(\begin{array}{c}
N \\
n_{1}
\end{array}\right)\left(\begin{array}{c}
N-n_{1} \\
n_{0}
\end{array}\right)
$$

When $K=1$, the number of regions of multiple equilibria is $\sum_{n=2}^{N}\left(\begin{array}{l}N \\ n\end{array}\right)$, i.e., all possible combinations of more than two outcomes. However, as illustrated in Table I, the number of regions for various values of $N$ and $K$ is generally far less from all the possible combinations. It means that the parametrization of the set of predicted choice probabilities is of a much lower dimension than one would have expected.

\section{[Include Table I]}

\subsubsection{The set of predicted choice probabilities}

We also define the subset of $S^{(K)}$ that contains one specific outcome $y_{j}^{(K)}$ as

$$
S_{j}^{(K)}=\left\{S \in S^{(K)}: y_{j}^{(K)} \in S\right\}
$$

Following Berry and Tamer (2007) and Galichon and Henry (2011), we can calculate the probability of observing outcome $y_{j}^{(K)}$. This probability depends on the parameter vector $\theta$ and on the unknown selection mechanism $\eta$ that selects equilibrium $y_{j}^{(K)}$ in the regions of multiple equilibria that predicts this outcome. More specifically,

$$
P_{j}^{(K)}(\theta, \eta)=\int_{U_{j}^{(K)}(\theta)} d F(\varepsilon ; \gamma)+\sum_{S \in S_{j}^{(K)}} \int_{\mathcal{R}_{S}^{(K)}(\theta)} \eta\left(y_{j}^{(K)} \mid \varepsilon ; \theta\right) d F(\varepsilon ; \gamma)
$$

where $U_{j}^{(K)}(\theta)$ is the region of $\varepsilon \in \mathbb{R}^{N}$ which uniquely predicts the outcome $y_{j}^{(K)}$. Let us denote by

$$
\Delta_{j}^{(K)}(\theta)=\int_{U_{j}^{(K)}(\theta)} d F(\varepsilon ; \gamma) \text { and } \Delta_{S}^{(K)}(\theta)=\int_{\mathcal{R}_{S}^{(K)}(\theta)} d F(\varepsilon ; \gamma) \text { for } S \in S^{(K)}
$$

Let $A(\theta)$ (resp. $B_{K}(\theta)$, for any $\left.K=0, \ldots, N\right)$ be the set of $P(\theta, \eta)$ (resp. $P^{(K)}(\theta, \eta)$ ) generated by the variation of $\eta$ in $\mathcal{E}$

$$
A(\theta)=\left\{P \in \mathbb{R}^{2^{N}}: \exists \eta \in \mathcal{E}, P=P(\theta, \eta)\right\}, B_{K}(\theta)=\left\{P^{(K)} \in \mathbb{R}^{d_{K}}: \exists \eta \in \mathcal{E}, P^{(K)}=P^{(K)}(\theta, \eta)\right\}
$$


Equation (3) is a parametrization of the sets $A(\theta)$ and $B_{K}(\theta), K=0, \ldots, N$. This parametrization is indexed by the regions $\mathcal{R}_{S}^{(K)}(\theta)$, counted in Table I.

\subsubsection{A characterization of the identified set}

Let $P_{0}=P\left(\theta_{0}, \eta_{0}\right)$ be the true choice probabilities generated by the true (unknown) parameter $\theta_{0}$ and the true (unknown) selection mechanism $\eta_{0} \cdot{ }^{11}$ The identified set $\Theta_{I}$ is defined as the collection of points $\theta$ such that $P_{0}$ can be rationalized with a selection mechanism

$$
\Theta_{I}=\left\{\theta \in \Theta: \text { such that } \exists \eta \in \mathcal{E}, P_{0}=P(\theta, \eta)\right\}
$$

The following is easily verified:

$$
\theta \in \Theta_{I} \text { if and only if } P_{0} \in A(\theta) \text {. }
$$

We therefore need to be more precise about the structure of $A(\theta)$ to be able to verify the second part. The following result holds:

Proposition $3 A(\theta)$ is a convex set of $\mathbb{R}^{2^{N}}$, and

$$
A(\theta)=B_{0}(\theta) \times B_{1}(\theta) \times B_{2}(\theta) \times \ldots \times B_{N}(\theta),
$$

where $B_{K}(\theta)$ is a convex set in $\mathbb{R}^{d_{K}}$.

The convexity of $A(\theta)$ is a general feature of an entry game and does not depend on our specification (see Beresteanu et al. (2011)). Its specific structure, i.e., the direct product of several components, comes from our specification in Equation (1) which ensures the unicity of the number of active firms in the regions of multiple equilibria. This structure simplifies some of the following results of this section.

Also, $B_{K}(\theta)$ is a point only when the number of active firms in equilibrium is 0 or $\mathrm{N}$, because there is no region of multiple equilibria involving these specific outcomes. Note that each $B_{K}(\theta)$ is strictly included ${ }^{12}$ in the cube, $\mathrm{Cub}_{K}$, defined by

$$
\Delta_{j}^{(K)}(\theta) \leq P_{j}^{(K)} \leq \Delta_{j}^{(K)}(\theta)+\sum_{S \in S_{j}^{(K)}} \Delta_{S}^{(K)}(\theta), \forall j=1, \ldots, d_{K}
$$

\footnotetext{
${ }^{11}$ We define $P_{0}^{(K)}=P^{(K)}\left(\theta_{0}, \eta_{0}\right), K=0, \ldots, N$ like in Equation (2).

${ }^{12} \mathrm{~A}$ visual illustration of the case with three players and $K=1$ is provided in the Supplementary Appendix.
} 
It comes from the fact that $\eta(\cdot)$ in Equation (3) lies between 0 and 1.

$\Theta_{I}$, the identified set, is not convex, but it can be characterized by verifying that a point, $P_{0}$, belongs to a convex set, $A(\theta)$. Using Proposition 3, we can decompose this condition into the following sub-conditions:

$$
P_{0} \in A(\theta) \text { iff } \forall K \in\{0,1, \ldots, N\}, P_{0}^{(K)} \in B_{K}(\theta)
$$

\subsection{The support function and a first selection of moment inequalities}

Following the convex literature, we introduce the support function of each convex set $B_{K}(\theta)$. This tool has, in particular, been used, in the set-identified literature, by Beresteanu and Molinari (2008) and Bontemps et al. (2012). It helps in generating the set of inequalities satisfied by $P_{0}$. We first recall what the support function of a convex set is and how it generates the inequalities that are the basis of our inference procedure. The support function of a convex set $A \subset \mathbb{R}^{d}$ is defined as:

$$
\delta^{*}(q ; A)=\sup _{x \in A} q^{\top} x
$$

for all directions, $q \in \mathbb{R}^{d}$. Its geometrical interpretation is illustrated in Figure 1. The support function of a convex set in a given direction locates the supporting hyperplane in this direction. For each direction $q$, it defines an inequality that is satisfied by any point of the convex set. The support function implicitly gathers all the inequalities that define the convex set into a single function. If the set is smooth, there is a continuum of such inequalities; if it is a polytope, only a finite number of inequalities is necessary to characterize the set. Kaido and Santos (2014) show that, when the set is convex, using the support function leads to an efficient estimator of the convex identified set.

Following Rockafellar (1970) and Proposition 3, the identified set is characterized by the following inequalities

$$
\begin{aligned}
\theta \in \Theta_{I} & \Longleftrightarrow P_{0} \in A(\theta) \\
& \Longleftrightarrow \forall q \in \mathbb{R}^{2^{N}}, q^{\top} P_{0} \leq \delta^{*}(q ; A(\theta)), \\
& \Longleftrightarrow \forall K, P_{0}^{(K)} \in B_{K}(\theta), \\
& \Longleftrightarrow \forall K, \forall q_{K} \in \mathbb{R}^{d_{K}}, q_{K}^{\top} P_{0}^{(K)} \leq \delta^{*}\left(q_{K} ; B_{K}(\theta)\right) .
\end{aligned}
$$

We now turn to the calculation of the support function of $B_{K}(\theta)$ for any $K$. Let $q_{K} \in \mathbb{R}^{d_{K}}$ be a given direction. We assume the following order among the coordinates of $q_{K}$ : $q_{i_{1}, K} \geq q_{i_{2}, K} \geq$ 
$\ldots \geq q_{i_{d_{K}}, K}$. We also partition $S^{(K)}$, the collection of subsets of outcomes with $K$ active firms in multiplicity, as follows: we denote $\mathcal{O}_{i_{1}}^{(K)}=S_{i_{1}}^{(K)}$, the elements of $S^{(K)}$ which contain the outcome $y_{i_{1}}^{(K)}$ and by $\mathcal{O}_{i_{2}}^{(K)}$ the subset of elements of $S_{i_{2}}^{(K)}$ that are not in $\mathcal{O}_{i_{1}}^{(K)}$, i.e., $S_{i_{2}}^{(K)} \backslash S_{i_{1}}^{(K)}$ and more generally $\mathcal{O}_{i_{j}}^{(K)}=S_{i_{j}}^{(K)} \backslash \cup_{k<j} S_{i_{k}}^{(K)}$, for any $j \leq d_{K}$. Note that the construction of the outcomes $\mathcal{O}_{j}^{(K)}$ 's is linked to the order of the components of $q_{K}$. We now provide a closed-form expression for the support function in this direction.

Proposition 4 Let $q_{K} \in \mathbb{R}^{d_{K}}$, and assume $q_{i_{1}, K} \geq q_{i_{2}, K} \geq \ldots \geq q_{i_{d_{K}}, K}$. The support function in the direction $q_{K}, \delta^{*}\left(q_{K} ; B_{K}(\theta)\right)$, is equal to:

$$
\delta^{*}\left(q_{K} ; B_{K}(\theta)\right)=\sum_{j=1}^{d_{K}} q_{j, K} \Delta_{j}^{(K)}(\theta)+\sum_{j=1}^{d_{K}} q_{i_{j}, K}\left(\sum_{S \in \mathcal{O}_{i_{j}}^{(K)}} \Delta_{S}^{(K)}(\theta)\right) .
$$

It is reached at the extreme point

$$
E_{i_{1}, i_{2}, \ldots, i_{d_{K}}}^{(K)}=\operatorname{vec}\left(\Delta_{1}^{(K)}(\theta)+\sum_{S \in \mathcal{O}_{1}^{(K)}} \Delta_{S}^{(K)}(\theta), \ldots, \Delta_{d_{K}}^{(K)}(\theta)+\sum_{S \in \mathcal{O}_{d_{K}}^{(K)}} \Delta_{S}^{(K)}(\theta)\right)
$$

Consequently, $B_{K}(\theta)$ is a polytope, and its vertices are included in the set of points $E_{i_{1}, i_{2}, \ldots, i_{d_{K}}}^{(K)}$ where the vector of indices $\left(i_{1}, \ldots, i_{d_{K}}\right)$ is any permutation of the vector of indices $\left(1,2, \ldots, d_{K}\right)$. $B_{K}(\theta)$ has at most $d_{K}$ ! vertices.

Each extreme point of $B_{K}(\theta)$, and therefore its support function, can be calculated from the knowledge of the non-zero values of $\Delta_{S}^{(K)}(\theta), S \in S^{(K)}$. This number of non-zero values is the number of multiple equilibria regions and we saw in Proposition 2 that this number is much smaller than $2^{d_{K}}-d_{K}-1$ (see table I). Consequently, the parametrization of $B_{K}(\theta)$ is numerically tractable for moderate values of $N$. Furthermore, each non-zero value $\Delta_{S}^{(K)}(\theta)$ can easily be calculated or simulated from the knowledge of the distribution of $\varepsilon$.

We can now extend this result to the calculation of the support function of the full set $A(\theta)$ for any direction $q \in \mathbb{R}^{2^{N}}$. We adopt the standard notation: $q=\operatorname{vec}\left(q_{0}, q_{1}, \ldots, q_{N}\right)$, where $q_{K}$ is the direction related to the set $B_{K}(\theta)$ (i.e., $q_{K} \in \mathbb{R}^{d_{K}}$ ) and $\operatorname{vec}(\cdot)$ denotes the vertical concatenation. 
Proposition 5 The support function of $A(\theta)$ in direction $q$ is equal to

$$
\delta^{*}(q ; A(\theta))=\sum_{K=0}^{N} \delta^{*}\left(q_{K} ; B_{K}(\theta)\right) .
$$

This results come from the specific characterization of $A(\theta)$ in Proposition 3. The last proposition, combined with Equation (7), is the basis of our inference procedure. It generates a continuum of inequalities that have to be satisfied for any parameter of the identified set. However, since all the $B_{K}(\theta)$ 's and, therefore, $A(\theta)$, are polytopes, it is necessary and sufficient to test the inequalities in a finite set of directions. We now explicit this set of directions, first for the $B_{K}(\theta)$ 's than for $A(\theta)$.

Let $\mathcal{Q}_{K}$ be the set of non-null directions of $\mathbb{R}^{d_{K}}$ with coordinates that are either one or zero. There are $2^{d_{K}}-1$ directions in $\mathcal{Q}_{K}$. The next proposition shows that it is sufficient to check the inequalities in $\mathcal{Q}_{K}$, for all $K$, to characterize the identified set.

\section{Proposition 6}

$$
\theta \in \Theta_{I} \Longleftrightarrow \forall K \in\{0,1,2, \ldots, N\}, \quad \forall q_{K} \in \mathcal{Q}_{K}, q_{K}^{\top} P_{0}^{(K)} \leq \delta^{*}\left(q_{K} ; B_{K}(\theta)\right)
$$

Remark We already mentioned that our specification ensures that the number of firms entering the market is constant among outcomes in multiplicity. As a result, the sets $B_{K}(\theta)$ belong to an hyperplane because the sum of the components of $P^{(K)}(\theta, \eta)$ is a constant which depends on $\theta$ only. If we wanted to characterize one $B_{K}(\theta)$ only, for one specific choice of $K$, we would need to consider

all the directions of $\mathcal{Q}_{K}$ combined with the direction $(-1,-1, \ldots,-1)$ to ensure the equality of the sum of all components. Here, due to the fact that we are considering $A(\theta)$, which is included in the simplex (the sum of all the probabilities is equal to 1$),{ }^{13}$ we don't need to consider this direction. As a matter of fact, if all the inequalities are satisfied with $(1, \ldots, 1)$ for all $K$, they are equalities and therefore are automatically satisfied for $(-1,-1, \ldots,-1)$.

Optimal transport, random sets or completion of the model Our approach consists in characterizing the set $A(\theta)$ (or, equivalently, the sets $B_{K}(\theta)$ ) through its support function and

\footnotetext{
${ }^{13}$ We thank one of the referees for highlighting this issue.
} 
extreme points. This is done after having completed the model with the unknown selection mechanism, $\eta(\cdot)$, and finding which selection mechanisms generate the extreme points. The geometric structure induced by the multiplicities allows us to exhibit the inequalities that are satisfied by any parameter of the identified set.

Galichon and Henry (2011) use the optimal transport theory and the notion of core determining class to generate the relevant inequalities that characterize sharply the identified set. Beresteanu et al. (2011) emphasize that an entry game is a model with convex predictions. They use random set theory and, in particular, the Aumann expectation considered in their paper is our set $A(\theta)$. Both methods are numerically challenging for a game with 6 players even when considering only pure strategy equilibria. Following Proposition 6, there are, at maximum, $\sum_{K=0}^{N}\left(2^{d_{K}}-1\right)$ inequalities. However, this number is very large when $N \geq 6$; we have more than 1 million of inequalities to check for 6 players.

Ciliberto and Tamer (2009) bound the sets $B_{K}(\theta)$ by the cubes $\mathrm{Cub}_{K}$ introduced above, which are easier to characterize. Their approach can handle games with a moderate number of players above 6, like our method, but bounding component by component makes the estimated set larger, and sharpness is not attained.

Fundamentally, whether one uses random set theory and the capacity functional, the optimal transport approach of Galichon and Henry (2011) or the approach presented in this paper, all these methods are intended to derive a sufficient set of inequalities satisfied by the parameters in a specific manner. Each method has its specificities. However, our approach allows us to go deeper into the geometric analysis of the set $A(\theta)$ and this is the objective of the next section.

\section{Using the geometry of $A(\theta)$ to select inequalities}

The convex set $B_{K}(\theta)$ can be characterized by at most $2^{d_{K}}-1$ inequalities. Due to its particular geometry, it may be the case that some of these inequalities are redundant. In this section, we present two strategies to reduce the number of inequalities. The first consists of calculating a core determining class introduced by Galichon and Henry (2011) and later used in Chesher and Rosen (2017). The second consists of exploiting further the geometry to propose a geometric selection procedure of the inequalities without having to evaluate all of them. 


\subsection{Deriving a core determining class of an entry game}

The core determining class introduced by Galichon and Henry (2011) yields to a collection of nonredundant moment inequalities that are sufficient to sharply characterize the identified set $\Theta_{I}$. We provide a characterization of the core determining class in an entry game from the geometric study of the multiplicity structure of the model. For the text to be self-contained, we borrow some definitions and concepts from Galichon and Henry (2011).

Definition 2 (Choquet capacity) A Choquet capacity $\mathcal{L}$ on the set $\mathcal{Y}$ is a set function $\mathcal{L}: C \subseteq$ $\mathcal{Y} \rightarrow[0,1]$ that is

- normalized, i.e., $\mathcal{L}(\emptyset)=0$ and $\mathcal{L}(\mathcal{Y})=1$, and

- monotone, i.e., $\mathcal{L}(C) \leq \mathcal{L}(B)$, for any $C \subseteq B \subseteq \mathcal{Y}$.

Definition 3 The smallest class $\Omega$ of subsets of $\mathcal{Y}$ is called core determining for the Choquet capacity $\mathcal{L}$ on $\mathcal{Y}$ if $\mathbb{P}(C) \leq \mathcal{L}(C)$ holds for all $C \in \Omega$; then, $\mathbb{P}(C) \leq \mathcal{L}(C)$ holds for all $C \subseteq \mathcal{Y}$.

The set $A(\theta)$ is characterized by its support function. Thus, we define the Choquet capacity for a subset $C_{K} \subseteq \mathcal{Y}_{K}$ as

$$
\mathcal{L}\left(C_{K}\right)=\delta^{*}\left(e_{C_{K}} ; B_{K}(\theta)\right)=\max _{\eta \in \mathcal{E}}\left(\sum_{j \mid y_{j}^{(K)} \in C_{K}} P_{j}(\theta, \eta)\right),
$$

where $e_{C_{K}} \in\{0,1\}^{d_{K}}$ with $\left(e_{C_{K}}\right)_{j}=1$ if $y_{j}^{(K)} \in C_{K}$ and 0 otherwise. For a collection of subsets $C=\left\{C_{K} \subseteq \mathcal{Y}_{K}: K \leq N\right\}$, the Choquet capacity is defined as $\mathcal{L}(C)=\sum_{k=0}^{N} \mathcal{L}\left(C_{K}\right) . \quad \mathcal{L}$ is monotone, as it is the sum of quantities that are positive and $\mathcal{L}(\mathcal{Y})=1$.

We define the concept of connectedness, which is useful for the exposition, introduced by Galichon and Henry (2011). For a subset $C_{K} \subseteq \mathcal{Y}_{K}$, we define the (undirected) graph generated by $C_{K}$ as $\Gamma_{C_{K}}=\left(C_{K}, E\right),{ }^{14}$ where there is an edge between two vertices if they are in multiplicity with eventually some additional outcomes that are only in $C_{K}$ (no outcome from $\mathcal{Y}_{K} \backslash C_{K}$ ). For any graph $\Gamma=(V, E)$, we say that $C \subseteq V$ is connected in the graph $\Gamma$ if there is a path of elements of $E$ connecting any pair of nodes of $C$.

\footnotetext{
${ }^{14}$ Recall that an undirected graph $\Gamma=(V, E)$ is a collection of vertices/nodes $V$ and edges/links $E$ that link these vertices. A graph $\Gamma$ is connected if any pair of vertices are connected in $\Gamma$.
} 
Definition 4 (Well connectedness) A subset $C_{K} \subseteq \mathcal{Y}_{K}$ is called well connected in $\mathcal{Y}_{K}$ if $\mathcal{Y}_{K} \backslash C_{K}$ is connected in the graph $\Gamma_{\mathcal{Y}_{K} \backslash C_{K}}$.

Note that $\mathcal{Y}_{K}$ is in multiplicity. Therefore, the graph $\Gamma_{\mathcal{Y}_{K}}$ is connected, and every $C_{K} \subseteq \mathcal{Y}_{K}$ is connected in the graph $\Gamma_{y_{K}}$. The notion of well connectedness extends the notion of connectedness by imposing restrictions on the complementary of $C_{K}$.

Note that the graph $\Gamma_{\mathcal{Y}}$ is not connected, as there is no multiplicity between $\mathcal{Y}_{K}$ and $\mathcal{Y}_{K^{\prime}}$, for $K \neq K^{\prime}$. Thus, $\Gamma_{\mathcal{Y}_{K}}$ is a component of $\Gamma_{\mathcal{Y}}{ }^{15}$ We collect all well-connected subsets of $\mathcal{Y}_{K}$ as

$$
\Omega_{K}=\left\{C_{K} \subseteq \mathcal{Y}_{K}: C_{K} \text { well connected in } \mathcal{Y}_{K}\right\}
$$

Galichon and Henry (2011) present some models in which the core determining class can be of much lower cardinality than $2^{|\mathcal{Y}|}$ by exploiting the monotonicity property in certain supermodular games. However, their approach does not provide a way to find a core determining class for a general entry game. Chesher and Rosen (2017) provide a sufficient condition to characterize a core determining class of set-identified models that can be written into what they call a generalized instrument variable model. Our next proposition provides a complete characterization of a core determining class for our entry model through a necessary and sufficient condition.

Proposition 7 A collection $\Omega$ of subsets of $\mathcal{Y}$ is core determining for $\mathcal{L}$ in (10) if and only if $\Omega=\left\{\Omega_{K}: K=0,1, \ldots, N\right\}$.

Subsection A.7 in the Supplementary Appendix provides an algorithm to construct the core determining class from Proposition 7. It is applied for the particular examples of $N=4$ and $K=2$ in Subsection A.7.2. However, it does not significantly reduce the number of non-redundant moment inequalities in our entry game. For example, when $N=6$ and $K=3$, it eliminates fewer than 30,000 inequalities from a total of $2^{20}-1=1,048,575$.

\subsection{A geometric selection procedure}

The core determining class is a useful concept because we eliminate redundant inequalities. However, it does not significantly reduce the number of inequalities in our entry game. We now present a

\footnotetext{
${ }^{15}$ Recall that for an undirected graph $\Gamma=(V, E)$, components of $\Gamma$ are subgraphs $\left\{H_{i}\right\}_{i=1}^{k}$ such that each $H_{i}$ is connected and $H_{i}$ is not connected to $H_{j}$ for $i \neq j$.
} 
geometric selection procedure that fully exploits the geometry of the sets $B_{K}(\theta)$. The procedure first selects the extreme point of the set that seems the closest to the vector $P_{0}^{(K)}$ and then evaluates only the inequalities associated with this extreme point, i.e., tests the directions that are the outer normal vector of the supporting hyperplanes of $B_{K}(\theta)$ at this point, and this, for each $K=0, \ldots, N$. Following Proposition 4, an extreme point is determined by an order in the coordinates (note that, a priori, two different orders could lead to the same physical point). The first part of the algorithm is intended to determine this order in a recursive manner by exploiting the position of $P_{0}^{(K)}$ with respect to the cube $\mathrm{Cub}_{K}$ which contains $B_{K}(\theta)$. We explain the steps in non-technical detail below and formalize the algorithm in Section A.8 of the Supplementary Appendix.

Local moment selection Our local moment selection procedure can be summarized as follows:

1. Determine the cube $\mathrm{Cub}_{K}$ that contains $B_{K}(\theta)$ by calculating the minimum and maximum of each coordinate. Then, determine which coordinate of $P_{0}^{(K)}$ is the furthest from the center of the cube.

2. Assume this is the $j^{\text {th }}$ coordinate.

(a) If it is on the maximum side, the extreme point is of type $E_{j, ?, \ldots, ?}^{(K)}(\theta)$, and we now have to determine the next component. To do so, we project $P_{0}^{(K)}$ on the face, and we repeat the previous calculation by taking into account that we are on the face that maximizes the $j^{\text {th }}$ coordinate.

(b) If it is on the minimum side, we know that our extreme point will be of the form $E_{?, \ldots, ?, j}^{(K)}(\theta)$, and we now have to determine the next component. To do so, we project $P_{0}^{(K)}$ on this face, and we repeat the previous calculation by taking into account that we are on the face that minimizes the $j^{\text {th }}$ coordinate.

3. Repeat the following steps until having found one order of coordinates.

4. Once the local extreme point $E_{i_{1}, i_{2}, \ldots, i_{d_{K}}}^{(K)}$ is determined, we can focus on the directions of the local supporting hyperplanes. Let the $d_{K}$ directions, $e_{i_{1}}, e_{i_{1}, i_{2}}, \ldots, e_{i_{1}, i_{2}, \ldots, i_{d_{1}-1}}, e_{i_{1}, i_{2}, \ldots, i_{d_{1}}}$, where the components are equal to 1 when the indices are subscripts of $e$ and 0 otherwise. This 
set of directions is included in the set of directions of the local supporting hyperplanes. Only checking these directions doesn't provide a sharp characterization of $B_{K}(\theta)$ unless $K=1$ or $N-1$ but, however, provides an important refinement with respect to the existing method of Ciliberto and Tamer (2009). An algorithm is provided in the Supplementary Appendix to derive the other directions to consider. ${ }^{16}$

Our procedure selects which moments among the $2^{d_{K}}-1$ are potentially binding without having to evaluate all of them. The selection is based on the spatial location of the point $P^{(K)}$ and exploits the geometry of the set $B_{K}(\theta)$. Proposition 8 shows that the procedure is sharp for $N=3$.

Proposition 8 Our local moment selection procedure provides a sharp characterization of the identified set for $N=3$.

However, it is difficult to prove sharpness with any number of players $N$ due to the difficulty of globally characterizing all the facets. We evaluate this procedure for $N=4$ in the Monte Carlo section and results highlight that we are sharp too.

\section{Estimation and inference}

Following the results derived above, we now adopt the approach developed in Beresteanu and Molinari (2008) and Bontemps et al. (2012) for testing a point in a convex set:

$$
\begin{aligned}
\theta \in \Theta_{I}(\mathbb{P}) & \Longleftrightarrow P_{0} \in A(\theta) \\
& \Longleftrightarrow \forall q \in \mathcal{G}, T_{\infty}(q ; \theta)=\delta^{*}(q ; A(\theta))-q^{\top} P_{0} \geq 0 \\
& \Longleftrightarrow \min _{q \in \mathcal{G}} T_{\infty}(q ; \theta) \geq 0 .
\end{aligned}
$$

$P_{0}$ is the true choice probability. The set of directions $\mathcal{G}$ is defined as

$$
\mathcal{G}=\bigcup_{K=0}^{N}\left\{\operatorname{vec}\left(0_{\sum_{i=0}^{K-1} d_{i}}, q_{K}, 0_{\sum_{i=K+1}^{N} d_{i}}\right): q_{K} \in \mathcal{G}_{K}\right\}
$$

\footnotetext{
${ }^{16}$ Intuitively, there are more facets at these extreme points because, due to the lower number of subsets in multiplicity, different orders of the indices $i_{1}, \ldots, i_{d_{K}}$ lead to the same point $E_{i_{1}, \ldots, i_{d_{K}}}^{(K)}(\theta)$.
} 
where, either $\mathcal{G}_{K}=\mathcal{Q}_{K}$ as defined in Proposition 6 or $\mathcal{G}_{K}=\Omega_{K}$ the core determining class characterized in Proposition $7 .{ }^{17}$ The set $\mathcal{G}$ collects successively all the directions needed to sharply characterize the identified set.

The test based on $T_{\infty}(\cdot)$ is infeasible because we do not observe $P_{0}$. We now characterize the feasible test statistic and its asymptotic distribution under the null and derive strategies to calculate the critical values. Throughout this section, we assume that we observe a sample of $M$ i.i.d. markets in which the same $N$ firms (known to the econometrician) compete.

\subsection{The asymptotic distribution of the test statistic}

Let $T_{M}(q ; \theta)$ be the empirical counterpart of $T_{\infty}(q ; \theta)$ :

$$
T_{M}(q ; \theta)=\delta^{*}(q ; A(\theta))-q^{\top} \hat{P}_{M}
$$

where $\hat{P}_{M}=\frac{1}{M} \sum_{m=1}^{M}\left[\mathbf{1}\left(Y_{m}=y_{1}\right), \ldots, \mathbf{1}\left(Y_{m}=y_{2^{N}}\right)\right]^{\top}$ is the empirical frequency vector. Under the assumption that the markets are i.i.d., we have:

$$
\sqrt{M}\left(\hat{P}_{M}-P_{0}\right) \underset{M \rightarrow \infty}{\stackrel{d}{\longrightarrow}} \mathcal{N}\left(0, \Sigma_{0}\right),
$$

where $\Sigma_{0}=\operatorname{diag}\left(P_{0}\right)-P_{0}\left(P_{0}\right)^{\top}$. Note that the only random part in $T_{M}(\cdot)$ comes from the estimation of the choice probabilities. Consequently, for $q$ and $\theta$ fixed, $T_{M}(q ; \theta)$ is asymptotically normal with variance $q^{\top} \Sigma_{0} q$. An empirical estimator, $\hat{\Sigma}$, can be used by plugging in $\hat{P}_{M}$ in place of $P_{0}$ in the expression of $\Sigma_{0}$.

In this section, we want our asymptotic result to be valid not only for the true probability but also uniformly in the neighborhood of the true probability. We impose the following uniform integrability condition:

Assumption 1 (Uniform integrability) The class $\mathcal{P}$ satisfies

$$
\lim _{\lambda \rightarrow \infty} \sup _{P \in \mathcal{P}} \sup _{j \in\left\{1, \ldots, 2^{N}\right\}} \mathbb{E}_{P}\left[\left(\frac{\mathbf{1}\left(Y=y_{j}\right)-\mu_{j}(P)}{\sqrt{\mu_{j}(P)\left(1-\mu_{j}(P)\right)}}\right)^{2} \mathbf{1}\left\{\left|\frac{\mathbf{1}\left(y_{j}\right)-\mu_{j}(P)}{\sqrt{\mu_{j}(P)\left(1-\mu_{j}(P)\right)}}\right|>\lambda\right\}\right]=0,
$$

\footnotetext{
${ }^{17}$ Observe that, if, for any $K, \tilde{q}_{K}=\operatorname{vec}\left(0_{\sum_{i=0}^{K-1} d_{i}}, q_{K}, 0_{\sum_{i=K+1}^{N} d_{i}}\right)$,

$$
\delta\left(\tilde{q}_{K} ; A(\theta)\right)=\delta\left(q_{K} ; B_{K}(\theta)\right) .
$$
}


where $\mu_{j}(P)=\mathbb{E}_{P}\left(Y=y_{j}\right)$.

Assumption UI ensures the uniform convergence of our test statistic over the class of probability distributions $\mathcal{P}$. This condition is satisfied over the class of probability distributions such that $\mu_{j}(P) \geq \varepsilon$ for each $j$ and some $\varepsilon>0$.

The literature on inference in partially identified models has largely focused on the construction of confidence regions $\mathcal{C}_{M}$. There is an open debate about whether one should cover any point of the identified set (and, in particular, the true value) or the identified set entirely. Unless stated otherwise, we are mainly interested in covering any point in the identified set with some pre-specified probability $1-\alpha$, i.e.,

$$
\liminf _{M \rightarrow \infty} \inf _{P \in \mathcal{P}} \inf _{\theta \in \Theta_{I}(P)} P\left(\theta \in \mathcal{C}_{M}\right) \geq 1-\alpha .
$$

Following Bontemps et al. (2012), our inference method is based on $T_{M}(q ; \theta)$, rescaled by $\sqrt{M}$ and normalized (see Chernozhukov et al., 2015):

$$
\xi_{M}(\theta)=\sqrt{M} \min _{q \in \mathcal{G}} \frac{T_{M}(q ; \theta)}{\sqrt{q^{\top} \hat{\Sigma} q}} .
$$

A point $\theta$ belongs to the confidence region $\mathcal{C}$ if the test based on $\xi_{M}(\theta)$ is not rejected. We now calculate the asymptotic distribution of the test statistics $\xi_{M}(\theta)$.

Proposition 9 Let $Q_{\theta}$ be the set of minimizers of $T_{\infty}(q ; \theta)$ in $\mathcal{G}$. Let $Z$ be a random vector of $\mathbb{R}^{2^{N}}$ distributed according to the normal distribution with variance $\Sigma_{0}$. We have

$$
\begin{cases}\xi_{M}(\theta) \underset{M \rightarrow \infty}{\stackrel{d}{\longrightarrow}} \min _{q \in Q_{\theta}} \frac{q^{\top} Z}{\sqrt{q^{\top} \Sigma q}} & \text { if } P_{0} \in A(\theta), \\ \xi_{M}(\theta) \underset{M \rightarrow \infty}{\stackrel{a . s}{\longrightarrow}}-\infty & \text { if } P_{0} \notin A(\theta),\end{cases}
$$

Under assumption UI, these results are uniformly valid over $P \in \mathcal{P}$.

Observe that the asymptotic distribution depends on $\theta$ only through $Q_{\theta}$. It is an important remark that we exploit in the next subsections to provide new critical values.

\subsection{A global bound approach}

We first propose conservative bounds. They have the advantage of being calculated simply and once for all for any $\theta$, thereby considerably simplifying the inference procedure. They can be used in a 
first step to locate the confidence region. This approach is based on the idea that we can trivially define two outer sets of $Q_{\theta}$ and, using these, bound the asymptotic distribution of $\xi_{M}(\theta)$. We thus have:

$$
Q_{\theta} \subset \mathcal{G} \subset \mathcal{R}
$$

where $\mathcal{R}$ is the set of all non null directions of $\mathbb{R}^{2^{N}}$. Consequently,

$$
\min _{q \in Q_{\theta}} \frac{q^{\top} Z}{\sqrt{q^{\top} \Sigma q}} \geq \min _{q \in \mathcal{G}} \frac{q^{\top} Z}{\sqrt{q^{\top} \Sigma q}} \geq \min _{q \in \mathcal{R}} \frac{q^{\top} Z}{\sqrt{q^{\top} \Sigma q}} .
$$

We denote respectively by $c(\mathcal{G}, \alpha)$ and $c(\mathcal{R}, \alpha)$, the $\alpha$ quantile of the bounding distributions. First, observe that

$$
\min _{q \in \mathcal{R}} \frac{q^{\top} Z}{\sqrt{q^{\top} \Sigma q}}=-\min \left\|Z^{*}\right\|,
$$

where $Z^{*}$ is distributed as a standard multivariate normal variable of dimension $2^{N}$. Second, we provide an algorithm in Section A.15 of the Supplementary Appendix to simulate the critical value $c(\mathcal{G}, \alpha)$ with a number of calculations polynomial in $N$. The confidence region is defined as the collection of points for which the test statistic $\xi_{M}(\theta)$ is above a given threshold:

$$
\mathcal{C}(c)=\left\{\theta \in \Theta: \quad \xi_{M}(\theta) \geq c\right\}
$$

Using the result of Proposition 9, we can show that the confidence regions built using our procedures have, asymptotically and uniformly over $\mathcal{P}$, the correct coverage rate for any point of the identified set.

Proposition 10 Under Assumption UI, the following holds:

$$
\liminf _{M \rightarrow \infty} \inf _{P \in \mathcal{P}} \inf _{\theta \in \Theta_{I}} P(\theta \in \mathcal{C}(c(\mathcal{R}, \alpha))) \geq \liminf _{M \rightarrow \infty} \inf _{P \in \mathcal{P}} \inf _{\theta \in \Theta_{I}} P(\theta \in \mathcal{C}(c(\mathcal{G}, \alpha))) \geq 1-\alpha
$$

Observe that because we bound the asymptotic distribution of both $\xi_{M}(\theta)$ uniformly for $\theta \in \Theta_{I}$, the confidence regions built from our global bounding strategy entirely cover the identified set. We therefore have the following stronger result:

Corollary 11 Under Assumption UI,

$$
\liminf _{M \rightarrow \infty} \inf _{P \in \mathcal{P}} P\left(\Theta_{I} \subset \mathcal{C}(c(\mathcal{R}, \alpha))\right) \geq \liminf _{M \rightarrow \infty} \inf _{P \in \mathcal{P}} P\left(\Theta_{I} \subset \mathcal{C}(c(\mathcal{G}, \alpha))\right) \geq 1-\alpha
$$




\subsection{A local bound approach}

The global approach above has a tremendous computational advantage because we calculate a critical value once. However, it is quite conservative. We now consider a new bound for the distribution of $\xi_{M}(\theta)$ based on a moderate deviation inequality for a self normalized sum used in Chernozhukov et al. (2014). It is based on the calculation of an upper bound on the number of facets of each set $B_{K}(\theta)$, which gives the highest possible number of binding moments.

\subsubsection{The number of facets of $B_{K}(\theta)$ at any extreme point}

We know from Proposition 4 , that $B_{K}(\theta)$ is included in an hyperplane of $\mathbb{R}^{d_{K}}$. It is due to the fact that the sum of the choice probabilities of all outcomes in $\mathcal{Y}_{K}$ is constant. Following the convex literature, an exposed face is the intersection between a supporting hyperplane and the convex set. Henceforth, we call facets of $B_{K}(\theta)$ at any extreme point all $d_{K}-1$ and $d_{K}-2$-faces containing this extreme point. Each facet is related to one supporting hyperplane which defines one non-redundant inequality.

From Proposition 4, the maximum number of extreme points of $B_{K}(\theta)$ is $d_{K} !$. We first consider the case in which $K=1$ as a benchmark (by symmetry, it also characterizes the case in which $K=N-1$ ) before considering the general case. Observe first that the geometry of a set $B_{K}(\theta)$ is the same as that of the set $B_{N-K}(\theta)$. We characterize the geometry of the sets $B_{1}(\theta)$ that will serve as a benchmark for considering the other sets.

Proposition 12 The convex set $B_{1}(\theta)$ has $d_{1}$ ! extreme points. Each of them is the intersection of $d_{1}$ supporting hyperplanes.

See the proof in the Supplementary Appendix. Following Proposition 2, we know that any subset of $\mathcal{Y}_{1}$ of cardinality greater than 2 corresponds to a multiple equilibria region. Consequently, $\Delta_{S}^{(1)}(\theta)$ for any subset $S \subseteq \mathcal{Y}_{1}$ is non-zero, and, following Proposition 4, any change in the order gives a different point $E_{i_{1}, i_{2}, \ldots, i_{d_{1}}}^{(1)}$.

For a general value of $K$, many collections of outcomes cannot be a prediction of a multiple equilibria region. Consequently, there are fewer than $d_{K}$ ! extreme points. Intuitively, different orders of the components of the direction $q_{K}$ may lead to the same physical extreme point (i.e., for 
example, $\left.E_{i_{1}, i_{2}, i_{3} \ldots, i_{d_{K}}}^{(K)}=E_{i_{1}, i_{3}, i_{2} \ldots, i_{d_{K}}}^{(K)}\right)$. More importantly, there are more than $d_{K}$ facets at these extreme points. The question is now to determine what are the facets at these specific extreme points and how many they are.

Proposition 13 Any extreme point of $B_{K}(\theta)$ for $1<K<N-1$ is the intersection of at most $2^{l_{\max }}+\left(d_{K}-l_{\max }-1\right) 2^{l_{\max }-1}$ supporting hyperplanes, where $l_{\max }$ is the maximum cardinality of a subset of $\left\{y_{1}^{(K)}, \ldots, y_{d_{K}}^{(K)}\right\}$ that cannot be in multiplicity. Furthermore, an algorithm is provided in the Supplementary Appendix to determine the facets at each extreme point $E_{i_{1}, i_{2}, i_{3} \ldots, i_{d_{K}}}^{(K)}$.

The result of Proposition 13 gives an upper bound on the number of facets at each extreme point and, therefore, on the number of binding moments. Observe that this number is exponentially smaller than the total number of inequalities $2^{d_{K}}-1$. The upper bound on the number of facets passing through any extreme point of $B_{K}(\theta)$ can be improved further on a case-by-case basis. Table II gives the maximum number of facets for $N=3$ to 6 .

\subsubsection{Using the maximum number of facets to calculate a new critical value}

The maximum number of facets of $A(\theta)$ at any extreme point is the sum of the maximum number of facets of each $B_{K}(\theta)$ calculated above. We call it $\mathcal{L}^{*}$.

Following Chernozhukov et al. (2014), we can use this number to provide a new critical value which is still conservative but much better than the ones derived in the global approach. Intuitively, the critical value calculated in the global approach assumes that all inequalities calculated from the direction in $\mathcal{Q}$ are binding whereas this new one only takes one of the existing extreme points as the worst case. The critical value is equal to

$$
c_{\mathcal{L}^{*}}(\alpha)=\frac{\Phi^{-1}\left(\alpha / \mathcal{L}^{*}\right)}{\sqrt{1-\Phi^{-1}\left(\alpha / \mathcal{L}^{*}\right)^{2} / M}}
$$

where $\Phi$ is the c.d.f. of the standard normal distribution, and $\Phi^{-1}$ its inverse. We have now the following result:

Proposition 14 Under Assumption UI,

$$
\liminf _{n \rightarrow \infty} \inf _{P \in \mathcal{P}} \inf _{\theta \in \Theta_{I}(P)} P\left(\theta \in \mathcal{C}\left(c_{\mathcal{L}^{*}}(\alpha)\right)\right) \geq 1-\alpha .
$$


Observe that the last procedure is not valid for covering the entire identified set because the set of minimizers $Q_{\theta}$, despite uniformly bounding its cardinal, varies with the point tested.

\section{Monte Carlo simulations}

We now evaluate the performance of the different testing procedures proposed in Section 4 in a simple game with $N$ players, with $N$ being equal to 3 and 4 . The profit function is equal to:

$$
\pi_{i m}=\beta+\alpha_{i} \sum_{j \neq i} a_{j m}+\varepsilon_{i m}
$$

where we assume that the term $\beta$ is the same across firms, and this is known to the econometrician. $\varepsilon_{i m}$, for $i=1, \ldots, N$, is drawn from a standard Normal distribution. We report the results for $M=1000$ independent markets. All the results displayed are based on 1000 replications, and the level of the tests is $\alpha=5 \%$.

\subsection{Experiments in a game with three players}

We consider a model with three symmetric players where $\beta=0.35$ and $\alpha_{1}=\alpha_{2}=\alpha_{3}=-0.4$. However, the econometrician only knows that $\beta$ is the same for all players. Consequently, the model is set-identified. The true selection mechanism gives an order of entry to firm $1,2,3$ in this order in the multiple equilibria regions. Thus, we have the following probabilities for the number of active firms in equilibrium: $P(K=(0,1,2,3))=(.048, .482, .435, .035)$. We also assume that the profit shocks are independent. For inference, we first compare three different approaches: "Bound" means that we only use the minimum and maximum of the probability of each possible outcome, as in (6), "Ineq" means that we test any point $\theta$ by considering the full set of directions proposed in Proposition 6. "Local" means that we apply our geometric selection procedure and only test the inequalities that define this local extreme point.

Critical values We display the results for the choice of two different critical values, $c(\mathcal{G}, \alpha)$ and $c_{\mathcal{L}^{*}}(\alpha)$ defined in subsections 4.2 and 4.3 (labeled "G" and "L" in the table). For the local approach, we also compare our results with the exact critical value which can be computed, in this case, due to the low dimensionality of the problem. 
Before turning to the results, we would like to compare our critical values with the ones which would have been computed while using the moment inequality literature. We consider the one of Andrews and Soares (2010) and its refinement by Romano et al. (2014). ${ }^{18}$

First, it would lead to an increasing amount of computer time. This essentially comes from the fact that the specific structure of the asymptotic distribution is not exploited and that the GMS procedure of Andrews and Soares (2010) has to first evaluate all inequalities, select them and bootstrap the test statistic for each point tested. The computation time drops from $11 \mathrm{~h}$ to $4 \mathrm{~s}$ on a single processor (for approximately 2.5 million points tested) when one exploits the geometry and computes the critical values proposed in the last section.

Second, the values are reported in Table III for two points, one on the boundary of the set $A(\theta)$ and one in the interior of the set. For small sample, the GMS procedures do not perform much better than the uniform approach suggested in our paper. One needs a very high sample size, greater than 3,000 to obtain better performances, and this is only true for the AS procedure for the interior point. Our local approach is, of course, much more accurate for testing a point located at the frontier, it is by construction more conservative for an interior point. Further results about the mean rejection rate across simulations for a sequence of points containing points from the identified set and outside this identified set are provided in the Supplementary Appendix and confirm the fact that our procedure works well.

Simulation results Table IV displays the results of our simulations. We report the information relative to the estimation of $\alpha_{1}, \alpha_{2}, \alpha_{3}$ and $\beta$. We test each point $\theta=\left(\alpha_{1}, \alpha_{2}, \alpha_{3}, \beta\right)$ on some predefined grid (defined below each table) and retain those that are not rejected by the testing procedures. The accepted points are then projected on each axis. We report the average of the minimum and maximum values across all simulations. It would be possible to consider the recent work of Kaido et al. (2018), who propose a procedure for subvector inference. However, here, we are mainly interested in comparing the different strategies. We also report the average number of points which pass the test normalized by the average number of points which pass the test in the local approach with the exact critical value. We call it "Vol. CR" in the Tables. This measure is a

\footnotetext{
${ }^{18}$ They are labeled respectively AS and RSW in the table.
} 
proxy for the relative sizes of the confidence regions.

We also propose different versions of the inequalities. There are many equivalent ways to characterize the identified set by a minimal set of inequalities and equalities. The small sample properties of the different testing strategies may be different. This is the reason why we compare the four equivalent sets of inequalities, ${ }^{19}$ which all sharply characterize $A(\theta)$. "Ineq1" considers the 16 directions in $\mathcal{Q}$ defined in Proposition 6; in "Ineq2", we replace the directions $(1,1,0)$ in $\mathcal{Q}_{K}$ for $K=1,2$ by the direction $(0,0,-1)$. It is based on the observation that testing the inequalities for $(1,0,0)^{\top},(1,1,0)^{\top}$ and $(1,1,1)^{\top}$ is equivalent to testing the inequalities for $(1,0,0)^{\top},(0,0,-1)^{\top}$ and $(1,1,1)^{\top}$. "Ineq3" considers the whole set of inequalities and equalities which define $B_{0}(\theta)$ to $B_{3}(\theta)$, dropping the equality related to $B_{2}(\theta)$, because of redundancy. There are 18 inequalities tested. Finally, "Ineq4" applies to Ineq ${ }_{3}$ the replacement of $(0,0,-1)$ by $(1,1,0)$ in $\mathcal{Q}_{K}$ for $K=1,2$.

\section{[Include Tables IV ]}

Obviously, a more conservative critical values leads to a larger confidence region. The conservative critical value $c(\mathcal{G}, \alpha)$ is not bad at all, even if a local analysis provides a better accuracy. There are some differences between the different sets of inequalities, and the bound approach seems competitive. It is worth noting that the DGP is in favor of the bounds because the independence between the profit shocks make the multiple regions very small.

The most efficient combination is "Inequ", whatever the choice of the critical value. The geometric selection procedure is conducted with the set of inequalities "Ineq" and the critical value $c_{\mathcal{L}^{*}}$. Unsurprisingly, it leads to the same outcome which is very close to the optimal one when one considers the exact critical value, a case difficult to consider when the number of players is higher.

Additional example We also report the results for a slight modification of the parameters ; it changes the accuracy of the bound approach. With $\beta=0.6, \alpha_{2}=-0.5$ and $\alpha_{1}=\alpha_{3}=-0.7$, we have the following probabilities for the number of active firms in equilibrium: $P(K=(0,1,2,3))=$ $(.021, .499, .464, .016)$. The model is also set-identified and the sizes of $B_{1}\left(\theta_{0}\right)$ and $B_{2}\left(\theta_{0}\right)$ are now larger. The results are displayed in Table V. The ratio between the bound approach and the

\footnotetext{
${ }^{19}$ The directions considered in these four cases are defined in subsection A.16.3 in the Supplementary Appendix.
} 
"sharp" inequality approach is more in favor of the sharp characterization. "Inequ" and our local procedure are still the two best procedures.

\section{[Include Tables V ]}

\subsection{A four player example}

Finally, we consider a four player example. We conduct a similar experiment than for the three player case with two different values of $\alpha_{i}, i=1, \ldots, 4$. We have $\beta=0.38, \alpha_{1}=\alpha_{4}=-0.35$ and $\alpha_{2}=\alpha_{3}=-0.2$. The order of entry of the firms in the multiple equilibria regions is purely random. Thus, we have the following probabilities for the number of active firms in equilibrium: $P(K=(0,1,2,3,4))=(.015, .237, .530, .207, .011)$. We assume that the econometrician knows that the true values of $\alpha$ are equal two by two. The model is therefore point-identified. The different set of directions considered are defined similarly as well as the critical values. We also report the results relative to our geometric selection procedure ("Local") in which we use $c_{\mathcal{L}^{*}}$ as the critical value. All the results are displayed in Table VI. First, the size of the confidence region calculated with the bound approach is much larger than the size of the same region calculated with our procedure. When the dimension of $\mathcal{Y}$ increases, the ratio between the volume of the cube which contains $A(\theta)$ and the volume of $A(\theta)$ increases too. A component wise bound approach selects much more points than a local approach (the volume ratio is close to 2). Like, for $N=3$, the same set of inequalities can be tested differently. Combining equalities and inequalities seem to be better (the volume ratio with respect to the most efficient combination are respectively equal to 1.5 for "Ineq1" and 1.3 for "Ineq3"). And, finally, following the results developed in this paper, a brute force algorithm would test 92 inequalities. A geometric selection procedure leads to the estimation of only 18 inequalities. It is still possible to compare this procedure with the brute force one for this value of $N$. Observe that they lead to the same size of the confidence region, highlighting the efficiency of our geometric selection procedure.

\section{[Include Table VI]}




\section{$6 \quad$ Extensions with explanatory variables}

We now generalize our approach to the case with discrete explanatory variables $Z \in \mathbb{R}^{l}$, whether $Z$ is genuinely discrete or discretized as in Ciliberto and Tamer (2009). Let the support of $Z$ be $\mathcal{Z}=\left\{z_{1}, \ldots, z_{d}\right\}$. The profit function in Equation (1) becomes

$$
\begin{aligned}
& \pi_{i m}=\beta_{i}+Z_{i m}^{\top} \gamma_{i}+\alpha_{i}\left(\sum_{j \neq i} a_{j m}\right)+\varepsilon_{i m}, \\
& a_{i m}=\mathbf{1}\left\{\pi_{i m}>0\right\} .
\end{aligned}
$$

All the discussion in the previous sections can now be generalized conditional on $Z_{m}=\left(Z_{1 m}, \ldots, Z_{N m}\right) \in$ $\mathcal{Z}^{N}$. For any realization $z_{m}$ of $Z_{m}$, the set $A_{z_{m}}(\theta)$ has exactly the same geometry than as described above. Let $P_{0}\left(z_{m}\right)$ be the true conditional choice probability vector. We obtain the following characterization of the identified set $(\theta$ represents all $\alpha, \beta, \gamma$ for all firms $i=1, \ldots, N)$ :

$$
\begin{aligned}
\theta \in \Theta_{I} & \Longleftrightarrow \forall z_{m} \in \mathcal{Z}^{N}, P_{0}\left(z_{m}\right) \in A_{z_{m}}(\theta) \\
& \Longleftrightarrow \forall z_{m} \in \mathcal{Z}^{N}, \forall q \in \mathcal{G}, T_{\infty}\left(q, z_{m} ; \theta\right):=\delta^{*}\left(q ; A_{z_{m}}(\theta)\right)-q^{\top} P_{0}\left(z_{m}\right) \geq 0 \\
& \Longleftrightarrow \min _{z_{m} \in \mathcal{Z}^{N}} \min _{q \in \mathcal{G}} T_{\infty}\left(q, z_{m} ; \theta\right) \geq 0
\end{aligned}
$$

The only difference is to add $\min _{z_{m} \in \mathcal{Z}^{N}}$ to the procedure, i.e., augment the space over which we take the minimum.

For the inference procedure, we first use a conditional frequency estimator to obtain the conditional choice probabilities. For each $y \in \mathcal{Y}$,

$$
\hat{P}_{M}\left(y \mid z_{m}\right)=\frac{\sum_{m=1}^{M} 1\left[Y_{m}=y\right] 1\left[Z_{m}=z_{m}\right]}{\sum_{m=1}^{M} 1\left[Z_{m}=z_{m}\right]} .
$$

We define the stacked vector as $\hat{P}_{M}\left(z_{m}\right)$. This estimator is uniformly consistent, asymptotically normal with a standard convergence speed.

The framework with conditioning variables is nearly the same as before. The only difference comes from the fact that we have to minimize the quantity $\inf _{q \in \mathcal{G}} T\left(q, z_{m} ; \theta\right)$ over the discrete space. The simulations of the critical values can be obtained equivalently. 


\section{Conclusion}

In this paper, we develop a new methodology to estimate games with multiple equilibria. The model may be set-identified, and belonging to this identified set is equivalent to testing whether the vector of choice probabilities belongs to a convex set. We characterize the full geometric structure of this convex set without adding any restriction on the selection mechanism in the regions with multiple equilibria. This approach has two advantages. First, it allows us to characterize all the moment inequalities that are necessarily satisfied for a parameter value to be in the identified set. Second, as the complexity of the problem grows exponentially with the number of players, we are able to propose an algorithm that geometrically selects the locally relevant moments, without having to evaluate all of them. The algorithm sequentially approaches the nearest vertex of the polytope, and it requires only the computation of the support function for a number of directions that is polynomial in $N$, the number of players.

This is a huge improvement in computational burden with respect to alternative methods, which need to numerically evaluate all the moments before deciding which ones are binding and which ones are not. This geometric understanding can easily be extended to other notions of equilibrium proposed in literature, e.g., two-level rationality and social interaction games. We also propose inference methods that for small and moderate sample sizes have better statistical properties than existing methods and offer a considerable advantage in terms of computational burden. Our methods exploit the specific structure of the test statistic, the variance of which does not depend on the parameter tested.

Many pending questions remain. First, entry games generally impose strong restrictions on the functional form of the profit function and on the distribution of the error terms. Adapting our procedure to more general settings (greater heterogeneity or semi-parametric forms) is high on the research agenda. Second, it would be worth investigating inference on subvectors, a question, that has recently been addressed in the partial identification literature, as in Kaido et al. (2018). Finally, we have thus far been agnostic about the (unobserved) selection mechanism, and we have used natural bounds. It would be worth investigating how restrictions on the selection mechanism could be incorporated into this framework. 


\section{Acknowledgements}

We gratefully acknowledge financial support from the Agence Nationale de Recherche ANR-05BLAN-0024-01. This research has also received financial support from the European Research Council under the European Community Seventh Framework Program FP7/2007-2013 grant agreement N_295298. We would like to thank the editor, the associate editor and two anonymous referees for helpful comments and participants at various conferences and seminars for helpful discussions. 


\section{Bibliography}

Andrews, D., and G. Soares (2010). Inference for Parameters Defined by Moment Inequalities Using Generalized Moment Selection. Econometrica 78 119-157.

Andrews, D., and X. Shi (2013). Inference Based on Conditional Moment Inequalities. Econometrica 81 609-666.

Aradillas-Lopez, A., and A. Rosen (2017). Inference in Ordered Response Games with Complete Information. Working paper.

Aradillas-Lopez, A., and E. Tamer (2008). The Identification Power of Equilibrium in Simple Games. J. Bus. Econ. Stat. 26(3) 261-283.

Bajari, P., H. Hong, and S. Ryan (2010). Identification and Estimation of Discrete Games of Complete Information. Econometrica 78 1529-68.

Beresteanu, A., and F. Molinari (2008). Asymptotic Properties for a Class of Partially Identified Models. Econometrica 76(4) 763-814.

Beresteanu, A., I. Molchanov and F. Molinari, (2011). Sharp Identification Regions in Models with Convex Moment Predictions. Econometrica 79 1785-1821.

Berry, S. (1992). Estimation of a Model of Entry in the Airline Industry. Econometrica 60 889-917.

Berry, S.,and P. Reiss (2007). Empirical Models of Entry and Market Structure, In: M. Armstrong and R. Porter, Editor(s), Handbook of Industrial Organization, Elsevier, 2007, Volume 3.

Berry, S., and E. Tamer (2007). Identification in Models of Oligopoly Entry, in Advances in Economics and Econometrics: Theory and Applications, Ninth World Congress, vol. 2, R. Blundell, W.K. Newey and T. Persson, eds., Cambridge Univ. Press.

Bjorn, P., and Q. Vuong (1984). Simultaneous equations models for dummy endogenous variables: a game theoretic formulation with an application to labor force participation. Working Paper, California Institute of Technology. 
Bontemps, C. and T. Magnac (2017). Set Identification, Moment Restrictions and Inference. Review of Economics and Statistics, 101, 146-159.

Bontemps, C., T. Magnac and E. Maurin (2012). Set Identified Linear Models. Econometrica 80 1129-1155.

Bresnahan, T., and P. Reiss (1991a). Entry and Competition in Concentrated Markets. Journal of Political Economy 99(5) 977-1009.

Bresnahan, T., and P. Reiss (1991b). Empirical models of discrete games. J. Econometrics 48(1-2) $57-81$.

Bugni, F. (2010). Bootstrap Inference in Partially Identified Models Defined by Moment Inequalities. Econometrica 78 735-754.

Canay, I. (2010). EL Inference for Partially Identified Models: Large Deviations Optimality and Bootstrap Validity. J. Econometrics 156 408-425.

Chernozhukov, V., D. Chetverikov, and K. Kato (2014) Testing many moment inequalities. arXiv:1312.7614

Chernozhukov, V., H. Hong, and E. Tamer (2007). Inference on Parameter Sets in Econometric Models. Econometrica 75 1243-1284.

Chernozhukov, V., E. Kocatulum and K. Menzel (2015). Inferences on sets in finance. Quantitative Economics 6 309-358.

Chesher A. and A. Rosen (2017). Generalized Instrumental Variable Model. Econometrica 85 959989.

Ciliberto, F., and E. Tamer (2009). Market Structure and Multiple Equilibria in Airline Markets. Econometrica 77 1791-1828.

Cleeren, K., M. Dekimpe, K. Gielens, and F. Verboven (2010). Intra- and Inter-format Competition among Discounters and Supermarkets. Marketing Science 29(3) 456-473. 
Galichon, A., and M. Henry (2011). Set Identification in Models with Multiple Equilibria. Rev. Econ. Stud. 78 1264-1298.

Grieco, P.(2014). Discrete games with flexible information structures: an application to local grocery markets. RAND Journal of Economics 45 303-340.

Gualdani C. (2019). An Econometric Model of Network Formation with an Application to Board Interlocks between Firms. TSE Working Paper 17-898, Toulouse School of Economics. Forthcoming Journal of Econometrics

Kaido, H., F. Molinari, and J. Stoye (2018). Confidence Intervals for Projections of Partially Identified Parameters. Working paper.

Kaido, H., and A. Santos (2014). Asymptotically efficient estimation of models defined by convex moment inequalities. Econometrica 82(1) 387-413.

Khan S. and E. Tamer (2010). Irregular Identification, Support Conditions, and Inverse Weight Estimation. Econometrica 78(6) 20212042.

Mazzeo M. (2002). Product Choice and Oligopoly Market Structure. Rand Journal of Economic $33(2) 1-22$.

Molchanov, I., and F. Molinari (2015). Applications of random set theory in econometrics. Annu. Rev. Econ. 6(1) 229-251.

de Paula A. (2013). Econometric Analysis of Games with Multiple Equilibria. Annu. Rev. Econ. 5 107-131.

Reiss, P. (1996). Empirical Models of Discrete Strategic Choices. American Economic Review 86 421-426.

Rockafellar, R.T. (1970). Convex Analysis. Princeton University Press.

Romano, J.P., and A.M. Shaikh (2008). Inference for Identifiable Parameters in Partially Identified Econometric Models. J. Statist. Plann. Infer. 138 2786-2807. 
Romano, J.P., and A.M. Shaikh (2010). Inference for the identified set in partially identified econometric models. Econometrica 78(1) 169-211.

Romano, J.P., and A.M. Shaikh (2012). On the Uniform Asymptotic Validity of Subsampling and the Bootstrap. Ann. Stat., 40(6) 2798-2822.

Romano, J., A. Shaikh, and M. Wolf (2014). A Practical Two-Step Method for Testing Moment Inequalities. Econometrica 82(5) 1979-2002.

Rosen, A. (2008). Confidence sets for partially identified parameters that satisfy a finite number of moment inequalities. J. Econometrics 146 107-117.

Sampaio R. (2007). Competition Between Low-Cost Carriers and Traditional Airlines: An Empirical Entry Model. TSE working paper.

Tamer, E. (2003). Incomplete simultaneous discrete response model with multiple equilibria. Rev. Econ. Stud. 70(1) 147-165. 


\section{Figures and Tables}

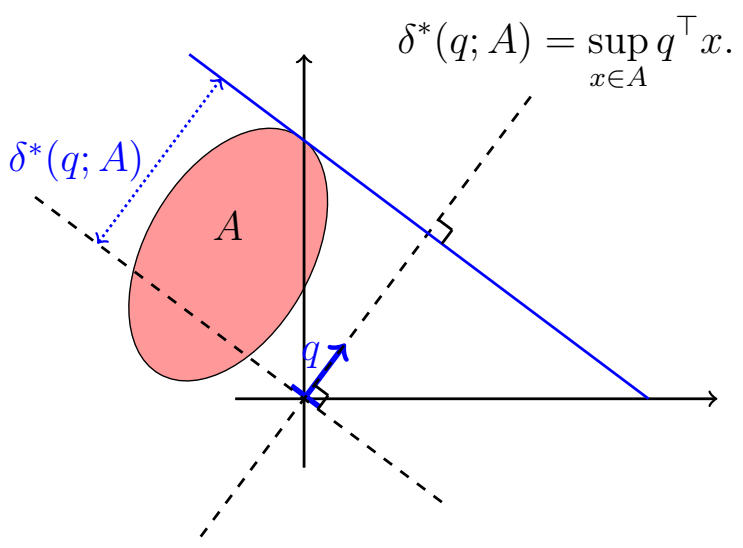

Figure 1: The support function

\begin{tabular}{ccccc}
\hline \hline$N$ & $K$ & $d_{K}$ & $\left|S^{(K)}\right|$ & $2^{d_{K}}-d_{K}-1$ \\
\hline 3 & 1 & 3 & 4 & 4
\end{tabular}

\begin{tabular}{ccccc} 
& 2 & 3 & 4 & 4 \\
\hline 4 & 1 & 4 & 11 & 11 \\
& 2 & 6 & 21 & 59
\end{tabular}

\begin{tabular}{lllll} 
& 3 & 4 & 11 & 11 \\
\hline 5 & 1 & 5 & 26 & 26
\end{tabular}

$\begin{array}{llll}2 & 10 & 71 & 1018\end{array}$

$\begin{array}{llll}3 & 10 & 71 & 1018\end{array}$

\begin{tabular}{ccccc} 
& 4 & 5 & 26 & 26 \\
\hline 6 & 1 & 6 & 57 & 57 \\
& 2 & 15 & 198 & 32761 \\
& 3 & 20 & 283 & 1048569 \\
& 4 & 15 & 198 & 32761 \\
& 5 & 6 & 57 & 57 \\
\hline \hline
\end{tabular}

Table I: Counting the number of multiple equilibria regions 


\begin{tabular}{|c|c|c|c|c|c|}
\hline & $B_{1}(\theta)$ & $B_{2}(\theta)$ & $B_{3}(\theta)$ & $B_{4}(\theta)$ & $B_{5}(\theta)$ \\
\hline \hline$N=4$ & 4 & 10 & 4 & & \\
\hline$N=5$ & 5 & 18 & 18 & 5 & \\
\hline$N=6$ & 6 & 52 & 136 & 52 & 6 \\
\hline
\end{tabular}

Table II: Upper bound on the number of facets at any extreme point for $N=4,5,6$.

\begin{tabular}{rccccc|ccccc}
\hline \multicolumn{4}{c|}{ Extreme point } & \multicolumn{5}{c}{ Interior point } \\
\hline & True & $G$ & $L$ & AS & RSW & True & $G$ & $L$ & AS & RSW \\
500 & -2.555 & -2.747 & -2.562 & -2.695 & -2.771 & -2.386 & -2.747 & -2.562 & -2.715 & -2.778 \\
1000 & -2.555 & -2.747 & -2.562 & -2.655 & -2.738 & -2.386 & -2.747 & -2.562 & -2.690 & -2.774 \\
2000 & -2.555 & -2.747 & -2.562 & -2.629 & -2.697 & -2.386 & -2.747 & -2.562 & -2.630 & -2.743 \\
3000 & -2.555 & -2.747 & -2.562 & -2.617 & -2.677 & -2.386 & -2.747 & -2.562 & -2.555 & -2.705 \\
10000 & -2.555 & -2.747 & -2.562 & -2.581 & -2.634 & -2.386 & -2.747 & -2.562 & -2.394 & -2.455 \\
\hline \hline
\end{tabular}

We compare the critical values of different procedures with the DGP of the set-identified model with 3 players, for two particular points tested. One point is an extreme point, i.e. 8 inequalities are binding in this point. One is an "interior" point, i.e. no inequality is binding but the ones related to the number of players.

Table III: Critical values for different procedures.

\begin{tabular}{|c|c|c|c|c|c|c|c|c|c|c|c|}
\hline \multirow[t]{13}{*}{ Type } & Test & $\begin{array}{l}\text { Crit. } \\
\text { value }\end{array}$ & $\begin{array}{c}\alpha_{1} \\
\min \end{array}$ & $\begin{array}{c}\alpha_{1} \\
\max \end{array}$ & $\begin{array}{c}\alpha_{2} \\
\min \end{array}$ & $\begin{array}{c}\alpha_{2} \\
\max \end{array}$ & $\begin{array}{c}\alpha_{3} \\
\min \end{array}$ & $\begin{array}{c}\alpha_{3} \\
\max \end{array}$ & $\begin{array}{c}\beta \\
\min \end{array}$ & $\begin{array}{c}\beta \\
\max \end{array}$ & $\begin{array}{l}\text { Vol. } \\
\text { CR }\end{array}$ \\
\hline & \multirow[t]{2}{*}{ Bound } & $G$ & -0.657 & -0.166 & -0.715 & -0.227 & -0.684 & -0.197 & 0.253 & 0.492 & 1.49 \\
\hline & & $L$ & -0.631 & -0.184 & -0.688 & -0.243 & -0.658 & -0.214 & 0.263 & 0.479 & 1.06 \\
\hline & \multirow[t]{2}{*}{ Ineq $_{1}$} & $G$ & -0.714 & -0.118 & -0.755 & -0.165 & -0.743 & -0.152 & 0.120 & 0.488 & 2.02 \\
\hline & & $L$ & -0.693 & -0.134 & -0.731 & -0.180 & -0.721 & -0.167 & 0.132 & 0.478 & 1.59 \\
\hline & \multirow[t]{2}{*}{ Ineq $_{2}$} & $G$ & -0.655 & -0.094 & -0.713 & -0.155 & -0.683 & -0.121 & 0.109 & 0.491 & 1.76 \\
\hline & & $L$ & -0.630 & -0.114 & -0.688 & -0.175 & -0.658 & -0.141 & 0.126 & 0.478 & 1.29 \\
\hline & \multirow[t]{2}{*}{$\mathrm{Ineq}_{3}$} & $G$ & -0.721 & -0.176 & -0.762 & -0.232 & -0.750 & -0.208 & 0.255 & 0.491 & 1.77 \\
\hline & & $L$ & -0.704 & -0.185 & -0.742 & -0.241 & -0.732 & -0.217 & 0.258 & 0.483 & 1.45 \\
\hline & \multirow[t]{2}{*}{ Ineq $_{4}$} & $G$ & -0.655 & -0.165 & -0.716 & -0.228 & -0.682 & -0.196 & 0.252 & 0.495 & 1.36 \\
\hline & & $L$ & -0.634 & -0.179 & -0.695 & -0.240 & -0.661 & -0.209 & 0.258 & 0.483 & 1.05 \\
\hline & \multirow[t]{2}{*}{ Local } & $L$ & -0.634 & -0.179 & -0.695 & -0.240 & -0.661 & -0.209 & 0.258 & 0.483 & 1.05 \\
\hline & & Exact & -0.630 & -0.181 & -0.692 & -0.242 & -0.658 & -0.212 & 0.259 & 0.481 & 1.00 \\
\hline
\end{tabular}

Note: the test statistic is $\xi_{M}(\theta)$ calculated on a grid of points $\left(\alpha_{1}, \alpha_{2}, \alpha_{3}, \beta\right):[-1.5 ; 0[$ for the alpha's with a tick of 0.03 and $[0 ; 1.2]$ for $\beta$ with a tick of 0.02 . The level is equal to $5 \%$. We display the mean across one thousand simulations of the minimum and maximum of each parameter. "Vol. CR" is the mean volume of the confidence regions normalized by the mean volume of the confidence regions computed with the local procedure with the exact critical value. The true values are $\beta=0.35$ and $\alpha_{1}=\alpha_{2}=\alpha_{3}=-0.4$, the selection mechanism gives priority to firm 1 than to firm 2 to enter in the multiple equilibria regions; the econometrician does not assume that the $\alpha^{\prime} s$ are the same. The model is set-identified.

Table IV: The confidence region - 3 players, set-identified case, 1000 markets. 


\begin{tabular}{|c|c|c|c|c|c|c|c|c|c|c|c|}
\hline \multirow[t]{13}{*}{ Type } & Test & $\begin{array}{c}\text { Crit. } \\
\text { value }\end{array}$ & $\begin{array}{c}\alpha_{1} \\
\min \end{array}$ & $\begin{array}{c}\alpha_{1} \\
\max \end{array}$ & $\begin{array}{c}\alpha_{2} \\
\min \end{array}$ & $\begin{array}{c}\alpha_{2} \\
\max \end{array}$ & $\begin{array}{c}\alpha_{3} \\
\min \end{array}$ & $\begin{array}{c}\alpha_{3} \\
\max \\
\end{array}$ & $\begin{array}{c}\beta \\
\text { min }\end{array}$ & $\begin{array}{c}\beta \\
\max \end{array}$ & $\begin{array}{l}\text { Vol. } \\
\text { CR }\end{array}$ \\
\hline & \multirow[t]{2}{*}{ Bound } & $G$ & -1.146 & -0.429 & -0.973 & -0.233 & -1.150 & -0.434 & 0.484 & 0.843 & 1.92 \\
\hline & & $L$ & -1.097 & -0.446 & -0.922 & -0.250 & -1.101 & -0.450 & 0.492 & 0.809 & 1.36 \\
\hline & \multirow[t]{2}{*}{ Ineq $_{1}$} & $G$ & -1.156 & -0.299 & -0.986 & -0.115 & -1.160 & -0.302 & 0.243 & 0.823 & 1.85 \\
\hline & & $L$ & -1.124 & -0.315 & -0.954 & -0.130 & -1.129 & -0.318 & 0.257 & 0.803 & 1.49 \\
\hline & \multirow[t]{2}{*}{ Ineq $_{2}$} & $G$ & -1.137 & -0.281 & -0.955 & -0.102 & -1.141 & -0.285 & 0.231 & 0.835 & 1.75 \\
\hline & & $L$ & -1.092 & -0.301 & -0.909 & -0.122 & -1.096 & -0.305 & 0.252 & 0.803 & 1.30 \\
\hline & \multirow[t]{2}{*}{$\mathrm{Ineq}_{3}$} & $G$ & -1.172 & -0.440 & -1.002 & -0.253 & -1.175 & -0.444 & 0.487 & 0.835 & 1.58 \\
\hline & & $L$ & -1.143 & -0.449 & -0.973 & -0.261 & -1.147 & -0.453 & 0.489 & 0.816 & 1.31 \\
\hline & \multirow[t]{2}{*}{ Ineq $_{4}$} & $G$ & -1.133 & -0.431 & -0.943 & -0.238 & -1.137 & -0.435 & 0.483 & 0.838 & 1.33 \\
\hline & & $L$ & -1.099 & -0.443 & -0.908 & -0.252 & -1.102 & -0.448 & 0.489 & 0.816 & 1.04 \\
\hline & \multirow[t]{2}{*}{ Local } & $L$ & -1.099 & -0.443 & -0.908 & -0.252 & -1.102 & -0.448 & 0.489 & 0.816 & 1.04 \\
\hline & & Exact & -1.093 & -0.446 & -0.904 & -0.254 & -1.097 & -0.451 & 0.491 & 0.814 & 1.00 \\
\hline
\end{tabular}

Note: the test statistic is $\xi_{M}(\theta)$ calculated on a grid of points $\left(\alpha_{1}, \alpha_{2}, \alpha_{3}, \beta\right):[-1.5 ; 0[$ for the alpha's with a tick of 0.03 and $[0 ; 1.2]$ for $\beta$ with a tick of 0.02 . The level is equal to $5 \%$. We display the mean across one thousand simulations of the minimum and maximum of each parameter. "Vol. CR" is the mean volume of the confidence regions normalized by the mean volume of the confidence regions computed with the local procedure. The true values are $\beta=0.6, \alpha_{2}=-0.5$ and $\alpha_{1}=\alpha_{3}=-0.7$ the selection mechanism gives priority to firm 1 than to firm 2 to enter in the multiple equilibria regions; the econometrician does not assume that $\alpha_{1}$ and $\alpha_{3}$ are the same. The model is set-identified.

Table V: The confidence region - 3 players, set-identified case, 1000 markets.

\begin{tabular}{clrrrrrrr}
\hline \hline Test & Crit. & $\alpha_{1}$ & $\alpha_{1}$ & $\alpha_{2}$ & $\alpha_{2}$ & $\beta$ & $\beta$ & Vol. \\
& value & $\min$ & $\max$ & $\min$ & $\max$ & $\min$ & $\max$ & CR \\
\hline Bound & $G$ & -0.602 & -0.232 & -0.455 & -0.062 & 0.253 & 0.682 & 2.60 \\
& $L$ & -0.576 & -0.240 & -0.430 & -0.072 & 0.260 & 0.648 & 2.04 \\
\hline Ineq1 $_{1}$ & $G$ & -0.573 & -0.242 & -0.421 & -0.095 & 0.176 & 0.631 & 2.21 \\
& $L$ & -0.542 & -0.254 & -0.394 & -0.106 & 0.197 & 0.593 & 1.55 \\
\hline Ineq $_{3}$ & $G$ & -0.575 & -0.247 & -0.423 & -0.097 & 0.259 & 0.633 & 1.80 \\
& $L$ & -0.547 & -0.258 & -0.399 & -0.108 & 0.271 & 0.600 & 1.30 \\
\hline Ineq4 & $G$ & -0.561 & -0.243 & -0.417 & -0.083 & 0.250 & 0.645 & 1.55 \\
& $L$ & -0.525 & -0.259 & -0.381 & -0.101 & 0.267 & 0.598 & 1.00 \\
\hline Local & $L$ & -0.526 & -0.258 & -0.381 & -0.100 & 0.267 & 0.598 & 1.00 \\
\hline
\end{tabular}

Note: the test statistic is $\xi_{M}(\theta)$ calculated on a grid of points $\left(\alpha_{1}, \alpha_{2}, \beta\right):[-1.5 ; 0[$ for the alpha's and $[0 ; 1.2]$ for $\beta$ with a tick of 0.01 . The level is equal to $5 \%$. We display the mean across one thousand simulations of the minimum and maximum of each parameter. "Vol. CR" is the mean volume of the confidence regions normalized by the mean volume of the confidence regions computed with the local procedure. The true values are $\beta=0.38, \alpha_{1}=\alpha_{4}=-0.35$ and $\alpha_{2}=\alpha_{3}=-0.2$, the order of entry of the firms in the multiple equilibria regions is random. The model is point-identified.

Table VI: The confidence region - 4 players, point-identified case, 1000 markets. 